\title{
Full Duplex-ing over Two Hops under Full Interference
}

\author{
Kudret Akçapınar \\ Sabancı University, FENS \\ Istanbul, TURKEY \\ Email: kudret@sabanciuniv.edu
}

\author{
Özgür Gürbüz \\ Sabanci University, FENS \\ Istanbul, TURKEY \\ Email: ogurbuz@sabanciuniv.edu
}

\begin{abstract}
In this paper, we study the optimization of transmission power in a two hop in-band full-duplex (IBFD) relaying scenario under full interference, i.e., when the source and destination nodes hear each other, which is highly likely in cellular or dense wireless networks. Considering imperfect selfinterference (SI) cancellation for IBFD operation at the relay node, we propose closed form solutions for calculating the power levels for the simultaneously transmitting nodes in an effort to achieve the maximum end-to-end throughput, and we derive an optimal power assignment policy. We compare the end-to-end throughput performance of IBFD relaying with proposed power assignment to that of traditional half-duplex (HD) relaying. Our results show the efficacy of power control for IBFD relaying, and show that the amount of performance improvement over traditional HD relaying depends on the level of SI suppression. More specifically, IBFD relaying has shown to be superior to HD relaying by up to $50 \%$ in the investigated scenarios.

Keywords - In-band full-duplex wireless, two hop communication, transmission power optimization, relaying, self-interference.
\end{abstract}

\section{INTRODUCTION}

Rapid proliferation of mobile devices and emergence of bandwidth intensive applications have led to an enormous increase in wireless traffic, which has resulted in a serious spectrum crunch. On the other hand, existing wireless spectrum has been mostly exploited, so new broadband technologies are required to allay the crisis. In-band full-duplex wireless communication has emerged as a promising candidate for future wireless communication systems to close the gap between actual and targeted capacity levels.

Until recently, a long-held taboo in wireless communication was that a radio cannot achieve simultaneous transmission and reception on the same frequency band, due to high selfinterference (SI) [1]. This assumption has been changed with the introduction and demonstration of in-band full-duplex (IBFD) communication, in works such as [2], [3]. It has been shown that via IBFD, capacity achieved with traditional halfduplex (HD) communication can be achieved occupying half of the total bandwidth only, and hence the spectral efficiency is doubled.

IBFD wireless technology offers a potential to double the spectral efficiency, enabling radios to perform simultaneous transmission and reception over the same frequency band. However, this is only true, when SI is completely suppressed. Because of the vigorous effect of the SI, decoding capability of the radios is degraded to a great extent. In order for a radio to perform successful IBFD communication, the SI must be reduced significantly, down to the noise level. This is especially challenging, since it is extremely stronger than the desired signal that the receive antenna is trying to pick up. On the other hand, by combination of successive advanced SI cancellation techniques [2], [3], IBFD operation has been made possible, and hence it has caught significant amount of attention recently.

In our previous work, [4], we have investigated the sum rate performance of bidirectional HD and IBFD communication to identify the conditions under which IBFD outperforms HD considering the effect of different system parameters. In [5], two hop communication with an IBFD relay is investigated and it is shown that the relay should employ power adaptation in order to maximize the system throughput. In [6], a modified version of the Dijkstra's algorithm for routing and a recursion based optimal transmit power allocation scheme for maximum end-to-end throughput are introduced for a multi hop IBFD network, assuming a simplified interference model, where only one hop interference is considered. In [7], a power controlled medium access control (MAC) scheme is proposed for forwarding packets in a cellular network scenario, where the relay is an access point (AP). In this scenario, the AP is assumed to relay (receive, decode-and-forward) packets in IBFD mode, and the uplink and downlink users hear each other, i.e., while an uplink user is transmitting to the $\mathrm{AP}$, it also interferes with the downlink user to which the AP is transmitting. The optimum transmission power levels for maximizing throughput are obtained for the uplink user and the AP via a heuristic solution and power control is implemented in the MAC protocol. However, none of the works mentioned above provides a closed form solution for the optimal transmission power for the full-interference scenario.

In [8], we have investigated the multi hop relaying problem by considering full interference across all nodes in the network. We have formulated and solved an optimization problem for the power levels in this problem and we have proven the advantage of considering full interference over considering only one hop interference [6]. In this paper, we consider a special case of this problem for two hops as we consider in-band full-duplex packet forwarding between source and destination nodes that hear each other. This is a typical 
scenario encountered in wireless dense networks. This relaying strategy is especially needed when source and destination node are not in the close proximity to maintain a successful communication, in which case relaying packets through an intermediate node in the network can potentially improve the end-to-end transmission rate. In the case of IBFD relaying, end-to-end throughput can be further improved depending on the interference resulted by IBFD operation. In order to achieve the maximum end-to-end throughput, transmission power of nodes should be optimally tuned. Differently from the existing works, employing a simple residual SI model, we derive closed form transmission power expressions for source and relay nodes, providing the maximum end-to-end throughput. We also compare the performance of IBFD relaying with that of HD relaying under different levels of residual SI. Via numerical simulations, we show that IBFD operation at the relay provides an improvement of up to $50 \%$ over HD in the investigated scenarios.

\section{System MODEL}

We investigate a two hop relaying scenario as depicted in Fig. 1. Here, node $A$ acts as a source node and aims to deliver its message to a destination node $B$ via an intermediate relay node $R$, which forwards the data from $A$ to $B$. As a real life example of this scenario, one can think of an access point (or base station) forwarding an uplink user's message to another user over the downlink channel. If the relay is $\mathrm{HD}$, then node $A$ first sends its packets to $R$. Once $A$ stops transmitting, $R$ employs in decode-and-forward (DAF) protocol for forwarding the packet to $B$. In case the relay is IBFD capable, it receives $i^{\text {th }}$ packet from $A$, while forwarding $(i-1)^{\text {th }}$ packet to $B$. It is realistic to assume that nodes $A$ and $R$ are power limited, such that $P_{A} \leq P_{A \max }$ and $P_{R} \leq P_{R \max }$, where $P_{A}$ and $P_{R}$ denote the actual transmit power at which nodes $A$ and $R$ operate, respectively, and $P_{A \max }$ and $P_{R \max }$ are the maximum transmit power of the respective nodes. As summarized in [9], FD communication is best suited for short range, such as femto cells because of the challenges of SI cancellation at higher transmit power levels. Therefore, we consider low transmission power and low noise levels, hence short range wireless communication scenarios. We assume that

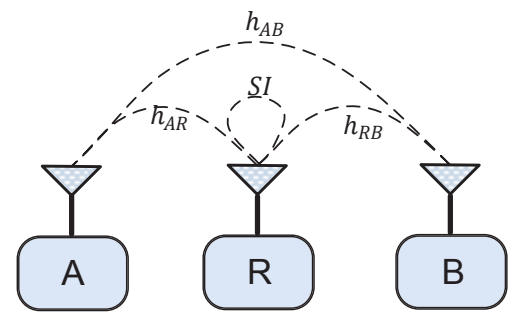

Figure 1: Two hop IBFD relaying model

all the nodes in the network have single antenna. If a node is operating in HD mode, its antenna is connected to either transmit RF chain or receive $R F$ chain by a means of a $T / R$ switch. For IBFD nodes, we assume a shared antenna design, where an antenna is shared by transmit and receive RF chains, similar to the design in [10].

In IBFD radios, since perfect isolation is not realizable, there is always a leakage from TX chain into RX chain, creating SI. We represent the effect of this SI, through its power via a simple mathematical model, as in [7], to facilitate the task of obtaining the closed form rate expressions. This SI model is defined as follows:

$$
I=\beta P_{T}
$$

Considering the generic relaying channel, where node $A$ is transmitting to node $B$ through node $R$, the received signals at nodes $B$ and $R$ can be written as follows:

$$
\begin{aligned}
& \mathbf{y}_{R}=h_{A R} \mathbf{x}_{A}+\mathbf{i}_{R}+\mathbf{w}_{R}, \\
& \mathbf{y}_{B}=h_{R B} \mathbf{x}_{R}+\mathbf{i}_{B}+\mathbf{w}_{B},
\end{aligned}
$$

where $h_{A R}, h_{R B}$ and $h_{A B}$ denote the Rayleigh channel coefficients of the respective links while $\mathbf{x}_{A}$ and $\mathbf{x}_{R}$ denote the vector of transmitted symbols. Similarly, $\mathbf{w}_{R}$ and $\mathbf{w}_{B}$ denote the AWGN noise with the same variance, $\sigma^{2}$. If node $R$ is operating in IBFD mode, $\mathbf{i}_{R}$ is the SI signal observed at node $R$ due to its own transmission and $\mathbf{i}_{B}$ denotes the inter node interference observed by node $B$ due to node $A$ 's transmission. We also define channel gains to be used in the proceeding sections such that $G_{\mathrm{AR}}=\left|h_{A R}\right|^{2}, G_{\mathrm{RB}}=\left|h_{R B}\right|^{2}$ and $G_{\mathrm{AB}}=\left|h_{A B}\right|^{2}$, all of which are exponentially distributed with a mean of $2 \sigma^{2}$ due to Rayleigh fading. In the case of HD operation, both interference terms are zero. Based on the received signals, the instantaneous rates from $A$ to $R, R_{A B}$ and $R$ to $B, R_{R B}$ are given by

$$
\begin{aligned}
& R_{A R}=\log \left(1+\Gamma_{R}\right), \\
& R_{R B}=\log \left(1+\Gamma_{B}\right) .
\end{aligned}
$$

where $\Gamma_{R}$ and $\Gamma_{B}$ denote the Signal to Noise Ratios (SNR) at nodes $R$ and $B$, respectively.

\section{Calculation of Achievable Rates}

\section{A. HD Achievable Rates}

If the relay is in HD mode, then links $A \longrightarrow R$ and $R \longrightarrow B$ must be active at alternating time slots, implying that $A$ and $R$ cannot transmit at the same time. For this reason, the flow of the information from source node, $A$ to destination node, $B$ occurs in two phases. First, $A$ transmits to $R$ and in the second phase $R$ forwards what it received from $A$ to $B$. SNRs during these phases are given by

$$
\Gamma_{R}=\frac{G_{\mathrm{AR}} P_{A}}{\sigma^{2}}, \quad \Gamma_{B}=\frac{G_{\mathrm{RB}} P_{R}}{\sigma^{2}} .
$$

In HD mode, the relay needs to devote different time slots to reception and transmission. Assuming that a fraction, $\tau$, of total communication time is dedicated to transmission of the source node, the rate achieved from node $A$ to node $R$ is obtained as:

$$
R_{A R}^{H D}=\tau \log \left(1+\Gamma_{R}\right)
$$


and the rate achievable over the link from $R$ to $B$ is given by

$$
R_{R B}^{H D}=(1-\tau) \log \left(1+\Gamma_{B}\right) .
$$

By optimizing over $\tau$, the end-to-end achievable average rate for $\mathrm{HD}$ relaying is obtained as

$$
R_{A B}^{H D}=\mathbf{E}\left[\max _{0 \leq \tau \leq 1} \min \left\{R_{A R}^{H D}, R_{R B}^{H D}\right\}\right] .
$$

Note that, in (7) increase in $\tau$ results in increase in $R_{A R}^{H D}$, yet decrease in $R_{R B}^{H D}$. As stated in [11], in this maxmin problem, optimal $\tau$, denoted by $\tau_{o p t}$ should satisfy $R_{A R}^{H D}=R_{R B}^{H D}$. Thus, $\tau_{\text {opt }}$ is given by

$$
\tau_{o p t}=\frac{\log \left(1+\Gamma_{B}\right)}{\log \left(1+\Gamma_{R}\right)+\log \left(1+\Gamma_{B}\right)}
$$

Note that, according to our system model, it is assumed that, the destination node $B$ can only hear (i.e., sense) but cannot decode node $A$ 's transmission. Hence node $B$ cannot benefit from the transmitted signal in the first phase and it can extract information only from relay's transmission as in [7], resulting in the rate expression in (7).

\section{B. IBFD Achievable Rates}

When the relay operates in IBFD mode, it is capable of transmitting and receiving at the same time. While receiving $i^{\text {th }}$ packet from the source node $A$, the relay can forward the previously received, $(i-1)^{t h}$ packet to the destination node $B$. As a result, links $A \longrightarrow R$ and $R \longrightarrow B$ are active simultaneously, unlike in HD relaying. This causes SI at the relay node, $R$. Meanwhile, the node $B$ receives $(i-1)^{t h}$ packet from $R$, also hearing from the transmission of the $i^{t h}$ packet from node $A$. Note that, node $B$ hears but it cannot decode $A$ 's transmission, and since node $B$ does not have multi packet reception capability, node $A$ 's transmission is treated as interference at node $B$. The Signal to Interference plus Noise Ratio (SINR) at nodes $R$ and $B$ can be obtained as:

$$
\begin{gathered}
\Gamma_{R}=\frac{G_{\mathrm{AR}} P_{A}}{\sigma^{2}+I_{R}}=\frac{G_{\mathrm{AR}} P_{A}}{\sigma^{2}+\beta P_{R}}, \\
\Gamma_{B}=\frac{G_{\mathrm{RB}} P_{R}}{\sigma^{2}+I_{B}}=\frac{G_{\mathrm{RB}} P_{R}}{\sigma^{2}+G_{\mathrm{AB}} P_{A}},
\end{gathered}
$$

where $I_{R}$ and $I_{B}$ denote the power of the interferences at nodes $R$, and $B$, representing the SI and inter node interference effects, respectively. For IBFD relaying, instantaneous rates of the two links are calculated as follows:

$$
\begin{aligned}
& R_{A R}^{I B F D}=\log \left(1+\Gamma_{R}\right), \\
& R_{R B}^{I B F D}=\log \left(1+\Gamma_{B}\right) .
\end{aligned}
$$

The average achievable end-to-end throughput from $A$ to $B$ given by

$$
R_{A B}^{I B F D}=\mathbf{E}\left[\max _{\substack{P_{A} \leq P_{A \max } \\ P_{R} \leq P_{R \text { max }}}} \min \left\{R_{A R}^{I B F D}, R_{R B}^{I B F D}\right\}\right] .
$$
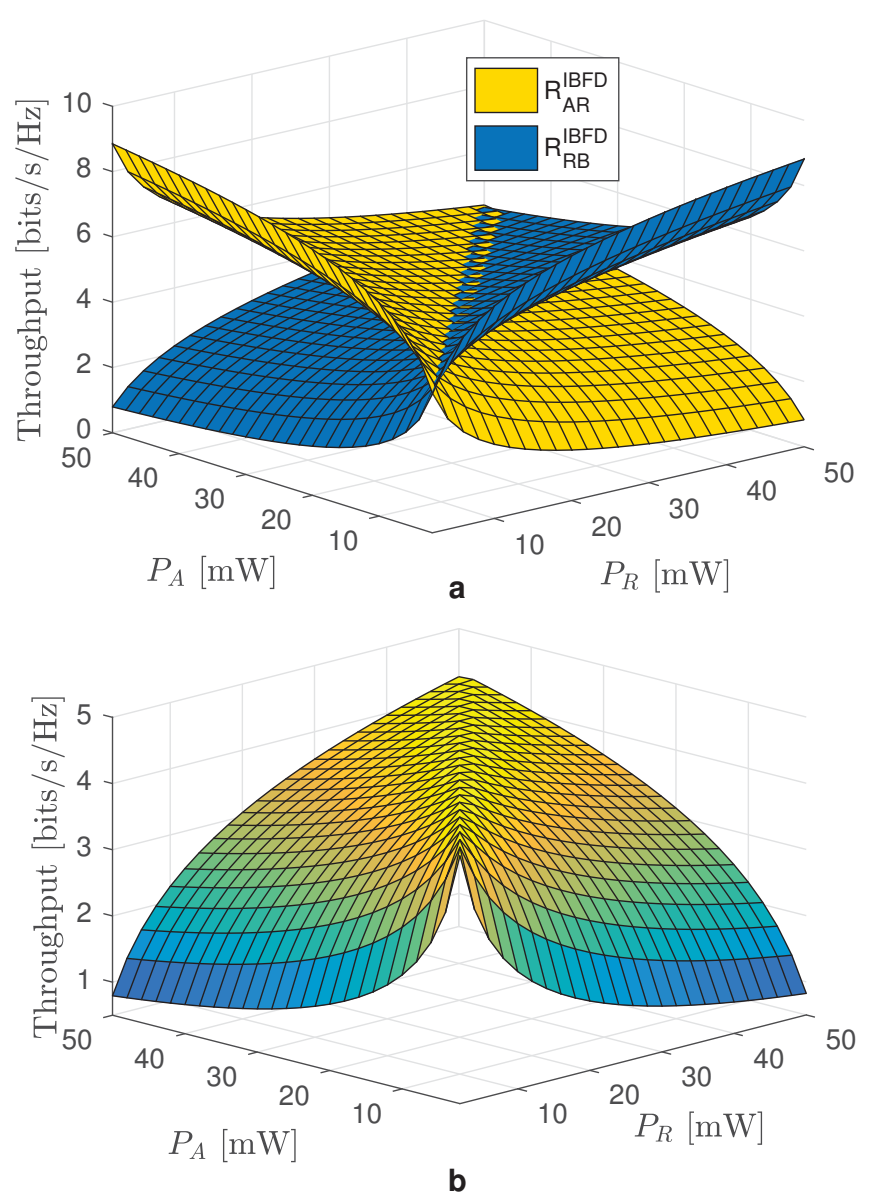

Figure 2: Throughput realization of a) $R_{A R}^{I B F D}$ and $R_{R B}^{I B F D}$ b) $R_{A B}^{I B F D}$ for $\beta=-80 d B$

Lemma : For maximum end-to-end throughput, achievable link rates $R_{A R}^{I B F D}$ and $R_{R B}^{I B F D}$ should be equal, which is consistent with the solution we obtained in [8] and the heuristic solution in [7].

The rate of link $A \longrightarrow R$ increases with $P_{A}$ and decreases with $P_{R}$ monotonically. Likewise, the rate of link $R \longrightarrow B$ increases with $P_{R}$ and decreases with $P_{A}$ monotonically. Additionally, the end-to-end rate is the minimum of these link rates. Therefore, end-to-end throughput is maximized when the link rates are equalized, which equivalently means, when SNRs at nodes $R$ and $B$ are equal. Assuming that $P_{A}$ and $P_{R}$ can be adjusted continuously, there has to be a pair $\left(P_{A}, P_{R}\right)$, which yields the equal link rates $\left(R_{A R}=R_{R B}\right)$.

The above lemma has been proven by the game theoretical approaches in earlier works on relaying such as [12], [13]. The game here is that there are two users who have the common objective, which is to maximize end-to-end throughput. Since the bargain parameters $\left(P_{A}\right.$ and $\left.P_{R}\right)$ are both continuous, there has to be a Nash equilibrium, and this equilibrium is reached, when the rates are equalized. By equating $\Gamma_{R}$ and $\Gamma_{B}$, we obtain the following equation 


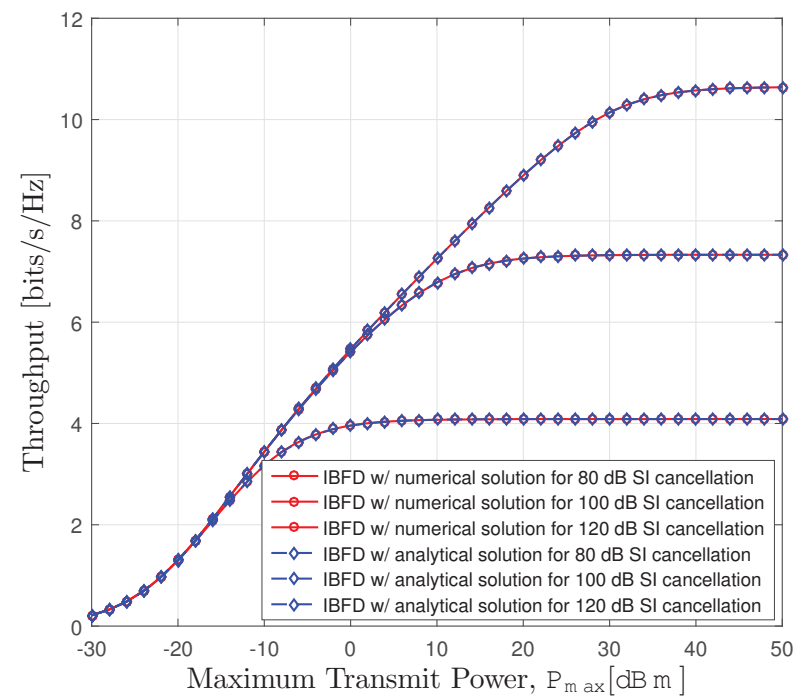

Figure 3: Validation of proposed closed form expressions for transmit powers with the numerical solution

$$
\frac{G_{\mathrm{AR}} P_{A}}{\sigma^{2}+\beta P_{R}}=\frac{G_{\mathrm{RB}} P_{R}}{\sigma^{2}+G_{\mathrm{AB}} P_{A}}
$$

Note that, equation (13) constitutes a quadratic equation for $P_{A}$ and $P_{R}$. Solving it with respect to $P_{A}$, we get the following roots:

$$
\begin{gathered}
P_{A_{1}}=\frac{-\sqrt{G_{\mathrm{AR}}} \sqrt{4 \beta G_{\mathrm{AB}} G_{\mathrm{RB}} P_{R}^{2}+4 \sigma^{2} G_{\mathrm{AB}} G_{\mathrm{RB}} P_{R}+\sigma^{4} G_{\mathrm{AR}}}-\sigma^{2} G_{\mathrm{AR}}}{2 G_{\mathrm{AB}} G_{\mathrm{AR}}} \\
P_{A_{2}}=\frac{\sqrt{G_{\mathrm{AR}}} \sqrt{4 \beta G_{\mathrm{AB}} G_{\mathrm{RB}} P_{R}^{2}+4 \sigma^{2} G_{\mathrm{AB}} G_{\mathrm{RB}} P_{R}+\sigma^{4} G_{\mathrm{AR}}}-\sigma^{2} G_{\mathrm{AR}}}{2 G_{\mathrm{AB}} G_{\mathrm{AR}}}
\end{gathered}
$$

Obviously, $P_{A_{1}}$ turns out to be negative. Since this is not possible, we choose $P_{A_{2}}$ as the solution to the equation (13). Substituting $P_{A}$ into $\Gamma_{R}$ or $\Gamma_{B}$, one can obtain the equal SNR levels $\Gamma_{R}=\Gamma_{B}$ as

$$
\frac{2 \sqrt{G_{\mathrm{AR}}} G_{\mathrm{RB}} P_{R}}{\sqrt{4 G_{\mathrm{AB}} G_{\mathrm{RB}} P_{R}\left(\beta P_{R}+\sigma^{2}\right)+\sigma^{4} G_{\mathrm{AR}}}+\sigma^{2} \sqrt{G_{\mathrm{AR}}}},
$$

which is a function of only one parameter, $P_{R}$. Our aim is now to find the best $P_{R}$ value, which will maximize $\Gamma_{R}$ or $\Gamma_{B}$ (Recall that $\left.\Gamma_{R}=\Gamma_{B}\right)$. Taking the first order derivative of (16) with respect to $P_{R}$, one can note that $\frac{d \Gamma_{R}}{d P_{R}} \geq 0$ since $\beta$ and all channel coefficients are positive. That is, $\Gamma_{R}=\Gamma_{B}$ is always increasing with respect to $P_{R}$, clearly for positive $P_{R}$ values. Hence, the solution for $P_{R}$ is on the boundary, implying that the relay should use its maximum power level i.e. $P_{R}=P_{R \max }$ in order to get the maximum achievable throughput. Node $A$, on the other hand, needs to adjust its transmission power according to (15). Hence, optimal power transmission levels are found as

$$
P_{A_{2}}=\frac{\sqrt{G_{\mathrm{AR}}} \sqrt{4 \beta G_{\mathrm{AB}} G_{\mathrm{RB}} P_{R}^{2}+4 \sigma^{2} G_{\mathrm{AB}} G_{\mathrm{RB}} P_{R}+\sigma^{4} G_{\mathrm{AR}}}-P_{R_{\text {max }}},}{2 G_{\mathrm{AR}}} .
$$

If the solution in (16) is not feasible for $P_{A}$, in other words, if $P_{A 2}$ found turns out to be greater than $P_{A \max }$, then $P_{A}$ is maximized, $P_{A}=P_{A_{\max }}$ and $P_{R}$ is obtained from the quadratic equation given in (13) following the same procedure as done for $P_{A}$

$$
P_{R}=\frac{\sqrt{G_{\mathrm{RB}}} \sqrt{4 \beta P_{A}^{2} G_{\mathrm{AB}} G_{\mathrm{AR}}+4 \beta \sigma^{2} P_{A} G_{\mathrm{AR}}+\sigma^{4} G_{\mathrm{RB}}}-\sigma^{2} G_{\mathrm{RB}}}{2 \beta G_{\mathrm{RB}}}
$$

We provide the rate surfaces as a function of transmit powers of the nodes in Fig. 2 for an easy visualization of the rate functions. We plot IBFD rate curves obtained by proposed analytic solution and numerical solution with respect to maximum transmit power for different level of SI suppression in Fig. 3, from which the proposed method can be validated. In Algorithm 1, we provide the pseudo code of the complete procedure for finding the optimal transmission power strategy for the investigated relaying scenario.

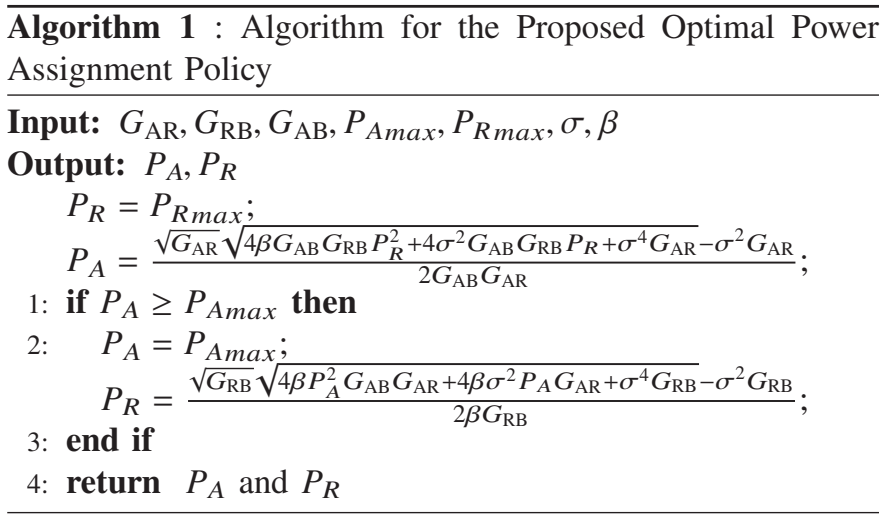

The proposed optimal power allocation algorithm requires channel state information (CSI) $h_{A R}, h_{R B}$ and $h_{A B}$, power budget of transmitting nodes, noise power at the receivers, $\sigma^{2}$ and SI cancellation parameter of IBFD relay, $\beta$. We assume that while power budget, noise power and SI cancellation parameter do not change with time, CSI of the links is assumed to remain same within a certain time frame (e.g. block fading). Therefore, it is necessary for the transmitting nodes to update CSI and hence transmission power policies according to Algorithm 1 prior to the transmission. It is also worthwhile to note that obtaining accurate channel state information requires design of a MAC protocol allowing the collection of CSI, which obviously results in extra overhead. Assuming availability of CSI, this work concentrates on power control in two hop IBFD relaying, so the presented results in this paper represent the performance upper bounds for two hop IBFD relaying. 


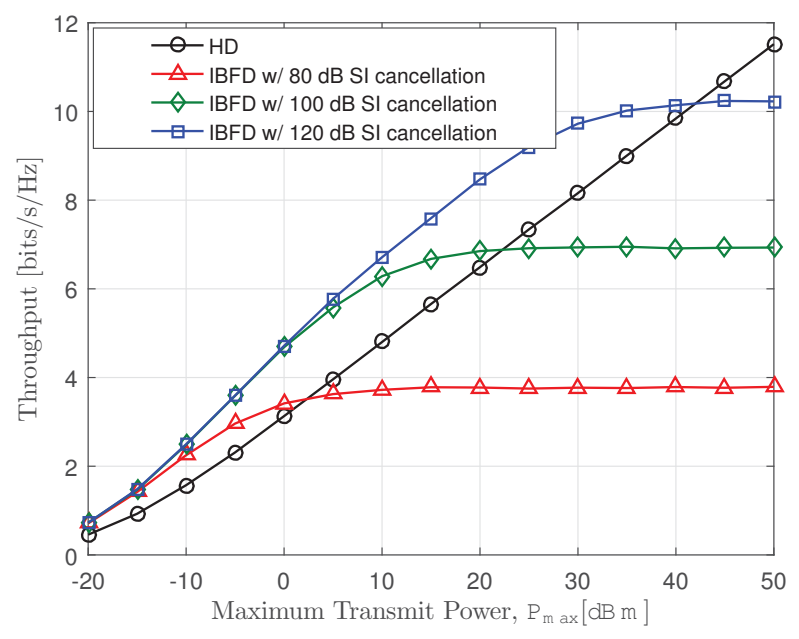

Figure 4: Throughput as a function of $P_{\max }$

\section{Numerical Results}

In this section, we compare the performance of HD and IBFD considering the system model described in Fig. 1 for several system parameters. In our simulations, noise variance, $\sigma^{2}$ and path loss exponent, $\alpha$ are taken to be $-90 \mathrm{dBm}$ and 4 , respectively. For IBFD scenario, we consider different levels of SI cancellation, such as $\beta=80 \mathrm{~dB}, \beta=100 \mathrm{~dB}$, and $\beta=120 \mathrm{~dB}$. Inter nodes distances $d_{A R}$ and $d_{R B}$ are both $50 \mathrm{~m}$ and $d_{A B}=100 \mathrm{~m}$.

In Fig. 4, the end-to-end throughput of the relaying system is shown as a function of maximum transmission power of relay. It is clear from Fig. 4 that IBFD outperforms HD when SI cancellation is strong enough, especially at low power regimes. The percentage gain of IBFD over HD is summarized in Fig. 5 for different values of $P_{\max }$ and $\beta$. As it can be seen from this Fig. 5, as long as IBFD radio has a strong SI cancellation capability, it always achieves a better performance than that of HD for the indicated distances. Fig. 5 also indicates that IBFD relaying is more convenient for low-power transmissions.

\section{CONCLUSion}

In this paper, we have focused on the optimization problem of transmission power of source and relay nodes in a basic one way two hop relaying scenario in order to achieve maximum end-to-end throughput. Considering a practical mathematical model for residual SI into account, we derive the closed form solutions for an optimal power assignment policy. Then, we evaluate the performance of IBFD relaying with power control, considering different levels of SI suppression through an example test scenario, suitable for IBFD operation (low transmission power, small distance). Our investigation over the effect of critical system parameters on the end-to-end throughput relaying performance has shown that IBFD relay outperforms HD relay as long as SI is significantly cancelled.

\section{ACKNOWLEDGEMENT}

This work was supported in part by TÜBİTAK Grant No: 113E222.

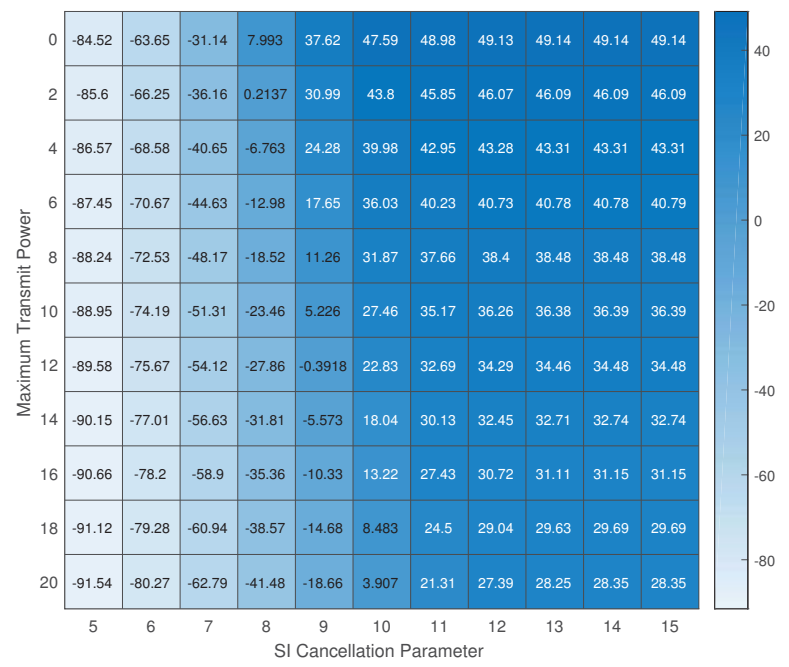

Figure 5: IBFD gain over HD for different $P_{\max }$ and $\beta$ values

\section{REFERENCES}

[1] A. Goldsmith, Wireless Communications. New York, NY, USA: Cambridge University Press, 2005.

[2] M. Duarte and A. Sabharwal, "Full-duplex wireless communications using off-the-shelf radios: Feasibility and first results," in 2010 Conference Record of the Forty Fourth Asilomar Conference on Signals, Systems and Computers, Nov 2010, pp. 1558-1562.

[3] J. I. Choi, M. Jain, K. Srinivasan, P. Levis, and S. Katti, "Achieving single channel, full duplex wireless communication," in Proceedings of the Sixteenth Annual International Conference on Mobile Computing and Networking, ser. MobiCom '10. New York, NY, USA: ACM, 2010, pp. 1-12.

[4] K. Akcapinar and O. Gurbuz, "Full-duplex bidirectional communication under self-interference," in Telecommunications (ConTEL), 2015 13th International Conference on, July 2015, pp. 1-7.

[5] T. Riihonen, S. Werner, and R. Wichman, "Hybrid full-duplex/halfduplex relaying with transmit power adaptation," IEEE Transactions on Wireless Communications, vol. 10, no. 9, pp. 3074-3085, Sept 2011.

[6] D. Ramirez and B. Aazhang, "Optimal routing and power allocation for wireless networks with imperfect full-duplex nodes," IEEE Transactions on Wireless Communications, vol. 12, no. 9, pp. 4692-4704, September 2013.

[7] W. Choi, H. Lim, and A. Sabharwal, "Power-controlled medium access control protocol for full-duplex wifi networks," Wireless Communications, IEEE Transactions on, vol. 14, no. 7, pp. 3601-3613, July 2015.

[8] K. Akcapinar and O. Gurbuz, "Optimal power control for full duplex wireless networks with full interference," in First International Balkan Conference on Communications and Networking (BalkanCom), 2017.

[9] A. Sabharwal, P. Schniter, D. Guo, D. Bliss, S. Rangarajan, and R. Wichman, "In-band full-duplex wireless: Challenges and opportunities," Selected Areas in Communications, IEEE Journal on, vol. 32, no. 9, pp. 1637-1652, Sept 2014.

[10] M. S. Amjad, H. Nawaz, K. Ozsoy, O. Gurbuz, and I. Tekin, "A LowComplexity Full-Duplex Radio Implementation With a Single Antenna," IEEE Transactions on Vehicular Technology, vol. 67, no. 3, pp. 22062218, March 2018.

[11] T. Riihonen, S. Werner, and R. Wichman, "Transmit power optimization for multiantenna decode-and-forward relays with loopback self-interference from full-duplex operation," in Signals, Systems and Computers (ASILOMAR), Nov 2011, pp. 1408-1412.

[12] Y. Shi, J. Wang, K. Letaief, and R. Mallik, "A game-theoretic approach for distributed power control in interference relay channels," Wireless Communications, IEEE Transactions on, vol. 8, no. 6, pp. 3151-3161, June 2009.

[13] Y. Jing and S. ShahbazPanahi, "Max-min optimal joint power control and distributed beamforming for two-way relay networks under per-node power constraints," Signal Processing, IEEE Transactions on, vol. 60, no. 12, pp. 6576-6589, Dec 2012. 